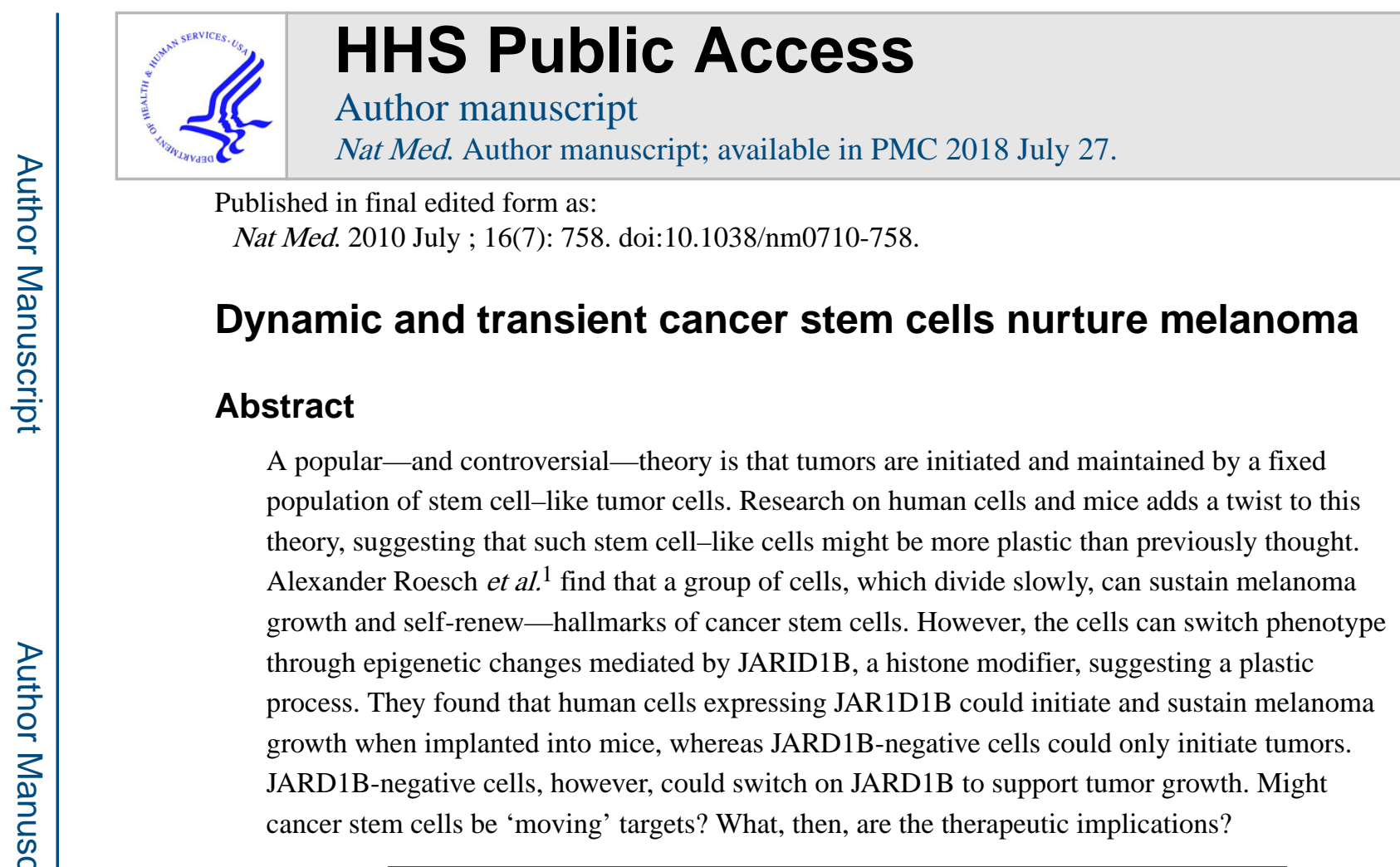

\title{
Robert Weinberg
}

The study by Roesch et al. ${ }^{1}$ indicates that the heterogeneity of a single melanoma can arise as a consequence of two processes - the formation of subpopulations of cells with distinct genetic states and the residence of cells in distinct epigenetic states, in this case slowly and rapidly proliferating cells, such as stem and non-stem cells in normal tissue.

Normal tissues may minimize the proliferation of stem cells to protect their genome integrity, which might be threatened by numerous divisions. Cancer-associated stem cells also tend to multiply more slowly than their non-stem cell progeny. In normal tissues, high mitotic activity is associated with progenitor cells, which function as intermediates between slowly proliferating stem cells and the fully differentiated descendents. Although not yet demonstrated, a similar state may operate within tumors.

Non-stem cell subpopulations may dedifferentiate into stem cells-a process suggested by indirect studies in normal and neoplastic cells-which would imply great plasticity and reversibility. The molecular regulators involved, if it occurs in both tissue types, remain obscure.

Roesch et al. ${ }^{1}$ do not directly identify these slow-cycling cells as cancer stem cells, but this may be a possible future direction, which would require extensive biological characterization of the cells, including tumor-seeding ability. Such identification might carry important clinical implications as cancer stem cells show increased resistance to conventional chemotherapy.

Member of Whitehead Institute for Biomedical Research and Professor of Biology, Massachusetts Institute of Technology, Cambridge, Massachusetts, USA. 


\section{David E Fisher}

Cell heterogeneity in solid tumors involves variability in marker expression, metabolic activity and survival tendency. The study by Roesch et al. ${ }^{1}$ sheds light on our understanding of melanoma cell heterogeneity and its effect on in vivo growth properties, which is incompletely understood.

Epigenetic regulation by JARID1B controls the longevity proliferative of melanoma cells in vivo. The fluid transition of cells from JARID1B positive to negative is fascinating, as it may help reconcile cellular heterogeneity with traditional tumor stem cell models. Whereas recent studies ${ }^{2}$ show the high tumor-initiating potential of nearly all melanoma cells, Roesch et al. ${ }^{1}$ suggest a separate role for these transient JARID1B-positive cells in sustaining tumor growth over time.

The researchers show inhibition of melanoma progression upon suppression of JARID1B, suggesting a possible therapeutic use for small molecules targeting JARD1B if such drugs are tolerated by normal tissues. Although it is unknown whether JARID1B has a similar effect in other malignancies, recent work ${ }^{3}$ showed a group of transient and drug-resistant cells in melanoma and nonmelanoma tumors that are dependent on the related histone demethylase JARID1A.

Director of the Melanoma Program, Massachusetts General Hospital, Harvard Medical School, Boston, Massachusetts, USA.

\section{Jeremy Rich}

Cellular plasticity permits cancer cells to adapt to environments to metastasize and develop therapeutic resistance. Roesch et al. ${ }^{1}$ show that melanomas take proliferative pauses to support long-term tumor maintenance. This potentially protects tumor cells from cytotoxic therapies, as quiescence is linked to resistance in leukemic stem cells. Chromatin regulation by JARID1B resembles the chromatin remodeling required for induced pluripotency-a process in which normal somatic stem cells gain an embryonic stem cell-like phenotypeindicating similarities between the plasticity of differentiation in cancer and normal cells during induced pluripotency.

This study ${ }^{1}$ indicates that the hierarchy driving the 'cancer stem cell hypothesis' may be malleable and not fated in one direction. Tumor hierarchy, and the epigenetics involved, may change with time and context adding to the complexity of cancer and limiting the development of 'silver bullets' against cancer stem cells.

Although this work was performed with human tissues, future work will also need in vivo human environments, as studies of hierarchy require greater clinical validation.

Chairman of Department of Stem Cell Biology and Regenerative Medicine, Lerner Research Institute, Cleveland, Ohio, USA. 


\section{References}

1. Roesch A, et al. A temporarily distinct subpopulation of slow-cycling melanoma cells is required for continuous tumor growth. Cell. 2010; 141:583-594. [PubMed: 20478252]

2. Quintana E, et al. Efficient tumour formation by single human melanoma cells. Nature. 2008; 456:593-598. [PubMed: 19052619]

3. Sharma SV, et al. A chromatin-mediated reversible drug-tolerant state in cancer cell subpopulations. Cell. 2010; 141:69-80. [PubMed: 20371346] 